\title{
Hierarchical Self-Assembly of Histidine-Functionalized Peptide Amphiphiles into Supramolecular Chiral Nanostructures
}

\author{
Meryem Hatip Koc, ${ }^{\dagger}$ Goksu Cinar Ciftci, ${ }^{\dagger}$ Sefer Baday, ${ }^{\dagger}$ Valeria Castelletto, ${ }^{\S \odot}$ Ian W. Hamley, ${ }^{\S}$ \\ and Mustafa O. Guler*, ${ }^{\dagger}, \|_{\mathbb{C}}$ \\ ${ }^{\dagger}$ Institute of Materials Science and Nanotechnology, National Nanotechnology Research Center (UNAM), Bilkent University, \\ Ankara, 06800 Turkey \\ ${ }^{\ddagger}$ Applied Informatics Department, Informatics Institute, Istanbul Technical University, Istanbul, 34469 Turkey \\ ${ }^{\S}$ Department of Chemistry, University of Reading, Whiteknights, Reading RG6 6AD, U.K. \\ "Institute for Molecular Engineering, University of Chicago, Chicago, Illinois 60637 United States
}

Supporting Information

ABSTRACT: Controlling the hierarchical organization of self-assembling peptide amphiphiles into supramolecular nanostructures opens up the possibility of developing biocompatible functional supramolecular materials for various applications. In this study, we show that the hierarchical self-assembly of histidine- (His-) functionalized PAs containing D- or L-amino acids can be controlled by both solution $\mathrm{pH}$ and molecular chirality of the building blocks. An increase in solution $\mathrm{pH}$ resulted in the structural transition of the His-functionalized chiral PA assemblies from nanosheets to completely closed nanotubes through an enhanced hydrogen-bonding capacity and $\pi-\pi$ stacking of imidazole ring. The effects of the stereochemistry and amino acid sequence of the PA backbone on the supramolecular organization were also analyzed by $\mathrm{CD}$, TEM, SAXS, and molecular dynamics simulations. In addition, an investigation of chiral mixtures revealed the differences between the hydrogen-bonding capacities and noncovalent interactions of PAs with D- and L-amino acids.

\section{INTRODUCTION}

Disordered constituents can spontaneously form stable organized patterns without any need for human intervention under equilibrium conditions in the self-assembly process. ${ }^{1}$ This natural process is important for the bottom-up fabrication of advanced functional nanostructures with the help of various noncovalent interactions such as van der Waals, ${ }^{2,3} \pi-\pi$ stacking, ${ }^{4,5}$ hydrogen-bonding, ${ }^{6}$ coordination-bonding, ${ }^{7}$ and electrostatic interactions. These noncovalent interactions guide the self-assembly of building blocks into supramolecular nanostructures with different morphologies ${ }^{8}$ including nanofibers, ${ }^{9,10}$ nanospheres, ${ }^{11,12}$ nanotubes, ${ }^{13-15}$ and nanoribbons. ${ }^{16}$

Among different self-assembling molecules, peptides are interesting building blocks for the construction of supramolecular assemblies because amino acids are the building elements of peptide molecules, which provide great diversity depending on their side-chain properties. ${ }^{17-19}$ Self-assembling peptide amphiphiles (PAs) are obtained through the conjugation of hydrophobic alkyl tails to hydrophobic and polar amino acid residues. ${ }^{20-22}$ The supramolecular organization of PA assemblies is modulated through molecular design exploiting the hydrogen-bonding properties of amino acid residues, ${ }^{23,24}$ as well as their hydrophobicity ${ }^{25,26}$ and molecular chirality. ${ }^{27}$ The structural organization of PA nanostructures can be also controlled by different external factors such as temperature, $^{28,29}$ photoirradiation, ${ }^{30,31}$ salt concentration, ${ }^{32}$ solvent effects, ${ }^{33}$ and $\mathrm{pH}^{34,35}$ owing to the dynamic nature of the self-assembly process. ${ }^{36}$

PA molecules can be functionalized with aromatic moieties including 9-fluorenylmethyloxycarbonyl (Fmoc), pyrene, and naphthalene as $\mathrm{N}$-terminal capping groups ${ }^{27,37-39}$ or aromatic amino acids such as diphenyl groups (-FF-) incorporated into the PA backbone ${ }^{40}$ to promote self-assembly using the directionality of $\pi-\pi$ stacking interactions ${ }^{41}$ along with hydrogen bonding and hydrophobicity. Furthermore, histidine(His-) containing PA building blocks have been developed to acquire $\mathrm{pH}$-sensitive control of the self-assembly behavior through the properties of the aromatic imidazole side chains. ${ }^{42}$ Branched PA molecules consisting of a tri-His headgroup and $\beta$-sheet-forming backbone were synthesized to develop $\mathrm{pH}$ switchable injectable hydrogels as tissue scaffolds. ${ }^{43}$ In another example, the design of $\mathrm{pH}$-sensitive oligo-His-containing PAs was presented to control the morphology of PA nanofibers and nanospheres at $\mathrm{pH}$ values between 6 and $7.5 .^{44} \mathrm{~A}$ PA

Received: April 12, 2017

Revised: July 14, 2017

Published: July 28, 2017 
incorporating a terminal histidine residue was shown to selfassemble into tape structures based on bilayers, with the number of bilayers within the nanostructures and the intermolecular interdigitation depending on the concentration. $^{45}$ The surfactant-like PA $\mathrm{A}_{6} \mathrm{H}$ shows self-assembled nanostructures that can be tuned by zinc chelation by the terminal histidine residue. ${ }^{46}$ The hexahistidine-containing PA $\mathrm{A}_{10} \mathrm{H}_{6}$ forms fibrils and can be tagged with nanogold using nickel nitrilotriacetic acid (Ni-NTA) coordination. ${ }^{47}$

In this study, we have designed histidine-functionalized PA building blocks containing either D- or L-amino acids ( $\mathrm{L}-\mathrm{VVHH}$, $\mathrm{D}-\mathrm{VVHH}, \mathrm{L}-\mathrm{FFHH}$, and D-FFHH). The functionalization of the PAs with a double-His headgroup $(\mathrm{HH})$ provided fine control over the supramolecular organization of the assemblies through a variety of noncovalent interactions under acidic, neutral, and basic conditions. In addition, the aromaticity differences between the $\beta$-sheet-forming regions were examined using different $\beta$-sheet-forming domains (-VVAG- and -FFAG-) in the peptide backbones. The supramolecular chirality of the PA assemblies is another important intrinsic property that can be manipulated using the chirality of the D- and L-amino acids. ${ }^{48}$ The effects of the amino acid chirality on the supramolecular organization were also analyzed using mixtures of different PA assemblies. In this article, we show the structural transition of the His-functionalized chiral PA assemblies from nanosheets to completely closed nanotubes with increasing $\mathrm{pH}$ and the effects of the molecular chirality of the building blocks on the supramolecular chiral organization.

\section{EXPERIMENTAL SECTION}

Materials. Fmoc and tert-butoxycarbonyl- (Boc-) protected amino acids, Rink Amide 4-methylbenzhydrylamine (MBHA) resin, and 2(1H-benzotriazol-1-yl)-1,1,3,3-tetramethyluronium hexafluorophosphate (HBTU) were purchased from NovaBiochem. All other solvents were purchased from Sigma-Aldrich.

Synthesis of Peptide Amphiphile by Solid-Phase Peptide Synthesis. All peptides were synthesized using standard Fmoc chemistry. All molecules, including those with a lauric acid tail, were constructed on Fmoc-Rink Amide MBHA resin. Amino acid coupling reactions were performed with 2 equiv of Fmoc-protected amino acid, 1.95 equiv of HBTU, and 3 equiv of $\mathrm{N}, \mathrm{N}$-diisopropylethylamine (DIEA) for $2 \mathrm{~h}$. Removal of the Fmoc protecting group was performed with $20 \%$ piperidine/dimethylformamide (DMF) solution for $25 \mathrm{~min}$. Cleavage of the peptides from the resin was carried out with a mixture of trifluoroacetic acid (TFA), triisopropyl silane (TIS), and $\mathrm{H}_{2} \mathrm{O}$ in a ratio of 95:2.5:2.5 for $2 \mathrm{~h}$. Excess TFA was removed by rotary evaporation. The remaining peptide was triturated with ice-cold diethyl ether, and the resulting white precipitate was freeze-dried. All peptides were purified by preparative high-performance liquid chromatography (prep-HPLC), and positive peptides were treated with $1 \mathrm{mM} \mathrm{HCl}$.

Liquid Chromatography-Mass Spectrometry (LC-MS). For structural and chemical analysis of the peptides, an Agilent Technologies 6530 Accurate-Mass quadrupole time-of-flight (QTOF) liquid chromatography-mass spectrometry (LC-MS) system with a Zorbax SB-C8 column were used. The concentration of the sample for LC-MS measurement was $0.5 \mathrm{mg} / \mathrm{mL}$. The solvents were water $(0.1 \%$ formic acid) and acetonitrile $(\mathrm{ACN})(0.1 \%$ formic acid). The LC-MS run for each sample was 30 min starting with $2 \%$ ACN and $98 \% \mathrm{H}_{2} \mathrm{O}$ for $5 \mathrm{~min}$. Then, the gradient of $\mathrm{ACN}$ reached $100 \%$ until 20 min. Finally, the ACN concentration was decreased to $2 \%$ for the last $5 \mathrm{~min}$. The solvent flow rate was $0.65 \mathrm{~mL} / \mathrm{min}$, and a sample volume of $5 \mu \mathrm{L}$ was injected.

Determination of Critical Aggregation Concentrations (CACs). The critical aggregation concentrations (CACs) of the PAs were determined using hydrophobic Nile Red (9-diethylamino-5benzo $[\alpha]$ phenoxazinone). Nile red exhibits an emission blue shift upon inclusion in a hydrophobic environment. The primary stock solution $(1.256 \mathrm{mM})$ of Nile red was prepared in ethanol $(0.4 \mathrm{mg}$ of Nile red $/ 1 \mathrm{~mL}$ of ethanol), and the stock solution was diluted to $78.125 \mu \mathrm{M}$ using ethanol (the total volume of the final Nile Red solution was $1.5 \mathrm{~mL}$ ). A $6.4 \mu \mathrm{L}$ volume of Nile Red in ethanol $(78.125$ $\mu \mathrm{M})$ was added to PA solutions $(996.8 \mu \mathrm{L})$ at different concentrations, and the mixtures were vortexed. The samples were then stored overnight at room temperature. In the spectrometer, the excitation wavelength was $550 \mathrm{~nm}$, and the emission spectra were collected between 580 to $750 \mathrm{~nm}$ using a Cary Eclipse fluorescence spectrophotometer.

Circular Dichroism (CD). A Jasco J-815 circular dichroism (CD) spectrophotometer was used for CD analysis. PA solutions [1\% (w/ $\mathrm{v})$ ] were prepared in water. The samples were gelled by the addition of $5 \mu \mathrm{L}$ of $0.1 \mathrm{M} \mathrm{NaOH}$ (for $\mathrm{pH} 7.4$ ) or $2.5 \mu \mathrm{L}$ of $1 \mathrm{M} \mathrm{NaOH}$ (for $\mathrm{pH}$ 10) and incubated overnight. The PA samples prepared at $\mathrm{pH} 4.5$ formed gels after overnight incubation without the addition of any $\mathrm{NaOH}$. After the gelation, the samples were diluted to a $0.5 \mathrm{mM}$ concentration using water. Spectra were measured from 300 to $190 \mathrm{~nm}$ with a 0.1 data pitch, a $100 \mathrm{~nm} / \mathrm{min}$ scanning speed, a $1 \mathrm{~nm}$ bandwidth, and a $4 \mathrm{~s}$ data acquisition time. All samples were measured in a $1 \mathrm{~mm}$ quartz cell. Averages of three measurements were used, and sensitivity was selected as the standard.

Transmission Electron Microscopy (TEM). Imaging of the peptides was achieved by transmission electron microscopy (TEM) (FEI, Tecnai G2 F30) at $100 \mathrm{kV}$. For PA nanofiber staining, uranyl acetate solution in water ( $2 \mathrm{wt} \%$ ) was used. The $2 \mathrm{mM}$ stock solution was diluted 40 times. Diluted samples were placed on a lacey-carboncoated copper grid. Ten milliliters of diluted sample solution was dropped on a grid and allowed to stand for $8 \mathrm{~min}$. The excess was removed by pipet. Then, $20 \mathrm{~mL}$ of $2 \mathrm{wt} \%$ uranyl acetate solution was put on a parafilm sheet. The grid was placed on the top of the drop with its top side down and held there for $5 \mathrm{~min}$. Stained grids were dried in the fume hood at room temperature overnight.

Small-Angle X-ray Scattering (SAXS). Small-angle X-ray scattering (SAXS) measurements were performed on beamline BM29 at the European Synchrotron Radiation Facility (ESRF), Grenoble, France. Solutions were loaded into the 96-well plate of a European Molecular Biology Laboratory (EMBL) BioSAXS robot and then injected by an automated sample exchanger into a quartz capillary (1.8-mm internal diameter) in the X-ray beam. The quartz capillary was enclosed in a vacuum chamber to avoid parasitic scattering. After the sample had been injected into the capillary and had reached the Xray beam, the flow was stopped during SAXS data acquisition. Beamline BM29 was operated with an X-ray wavelength of $\lambda=1.03 \AA$ $(12 \mathrm{keV})$. The images were captured using a PILATUS $1 \mathrm{M}$ detector, and data processing was performed using the dedicated beamline software ISPYB (Information System for Protein Crystallography: Beamlines) (BM29). Because of the strong anisotropy of the observed SAXS patterns for some samples (discussed below), in some cases, remasking and rebinning of the data was necessary to generate satisfactory radially averaged intensity profiles.

Computational Experiments. The dynamics of PA nanostructures were investigated by all-atom explicit-solvent molecular dynamics (MD) simulations. The PA nanostructures were modeled as cylindrical nanofibers, and these structures were used as initial structures for the simulations. Each PA nanofiber system was built using 19 layers composed of 12 PA molecules each, and each system contained 228 PA molecules. The starting configuration for each PA nanofiber was based on the results of a previous study, which suggested that 19 layers with 12 PAs in each layer configuration gives rise to the most stable configuration for PAs having a similar length consistent with experimental results. ${ }^{49}$ Each layer was built by placing 12 PAs with $30^{\circ}$ angles between them. Adjacent layers were assembled with a 5- $\AA$ separation and $15^{\circ}$ angle rotation (Figure S8). The PA nanofibers were solvated with water molecules modeled using TIP3, and $\mathrm{Na}^{+}$and $\mathrm{Cl}^{-}$ions were added to reach a salt concentration of $0.15 \mathrm{M}$. The resulting simulation-system boxes comprised approximately 120000 atoms. MD simulations of the PA nanofibers were performed using the NAMDprogram (version 2.9) with the CHARMM force field. ${ }^{50,51}$ 


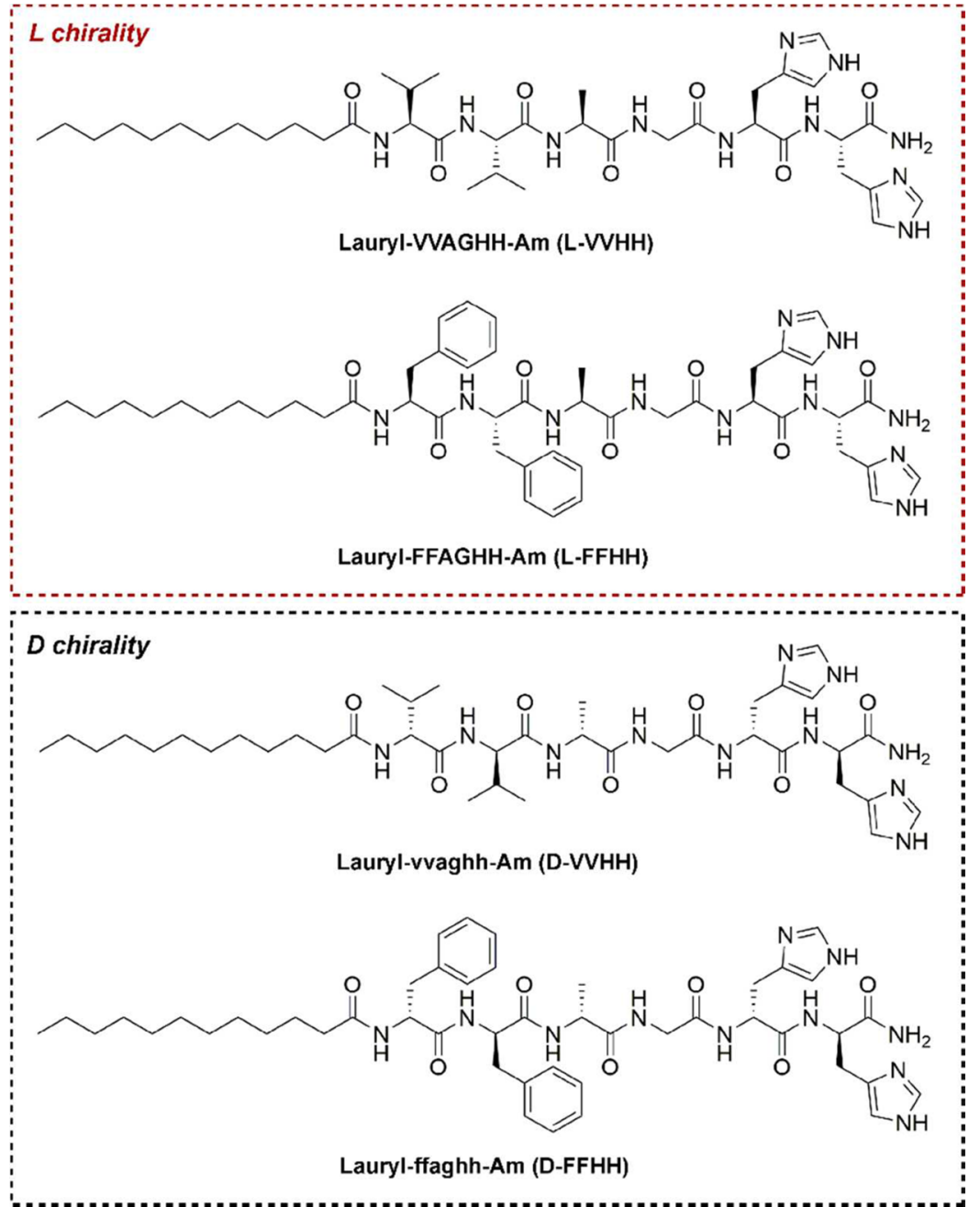

Figure 1. Chemical representation of histidine-functionalized peptide amphiphile building blocks with L and D chiralities.

Prior to production simulations, simulation systems were minimized with 1000 minimization steps. Then, 50-ns production simulations were carried out for each PA nanofiber system at $1 \mathrm{~atm}$ pressure and $310 \mathrm{~K}$ temperature. Electrostatic interactions were calculated using the particle-mesh Ewald method with a grid spacing. ${ }^{52}$ The cutoff for van der Waals interactions was taken as $12 \AA$, with a switching function after $10 \AA$. Simulation trajectories were integrated with a time step of 2 $\mathrm{fs}$, with all interactions calculated at every time step. Atomic coordinates were collected every 10 ps. The analyses were applied to the last $10 \mathrm{~ns}$ of each trajectory. Hydrogen bonds were calculated using the program CPPTRAJ. ${ }^{53}$ Calculations of nonbonded interaction energies for the simulation trajectories were carried out using the VMD program. ${ }^{54}$

Sample Preparation for Chiral Mixtures. For the preparation of chiral mixtures, the PA powders were initially dissolved in hexafluoroisopropanol (HFIP), which is a strong H-bond-donating solvent, to prevent individual self-assembly of the PA molecules prior to chiral mixing. The PA solutions (with D or L chirality) prepared in HFIP were mixed at $100 \%, 75 \%, 50 \%, 25 \%$, and $0 \%$ molecular ratios. Then, HFIP was removed under a vacuum, and the chiral mixture powders were resuspended in water at a PA concentration of $0.5 \mathrm{mM}$. The $\mathrm{pH}$ was adjusted using $\mathrm{NaOH}$ solution.

\section{RESULTS AND DISCUSSION}

Design, Synthesis, and Self-Assembly of the Histidine-Functionalized PA Building Blocks. Amino acids exhibit a great diversity in their side chains including charge, hydrophobicity, polarity, and aromaticity. ${ }^{18}$ Histidine (His) contains an aromatic imidazole side chain that plays an important role in protein-protein interfaces and catalysis because of its capacity for multiple interactions with other molecules through cation $-\pi$ (when His is protonated, $\mathrm{His}^{+}$), $\pi-\pi$ stacking, hydrogen $-\pi$, hydrogen-bonding, and coordination interactions with metallic cations. ${ }^{55}$

PAs consisting of a hydrophobic alkyl tail and aliphatic or aromatic amino acid domains were functionalized at the $\mathrm{N}$ terminus with double-histidine head groups (Figure 1). The integration of two histidines within the PAs resulted in selfassembling building blocks with multiple interaction capabilities at different $\mathrm{pH}$ values. Two different $\beta$-sheet-forming domains, namely, -VVAG- and -FFAG-, were also conjugated to the molecules so that hydrogen-bonding capacities of aliphatic and aromatic side chains could be studied. In addition to the differences in amino acid residues, the PA molecules were built with either L or D chirality to reveal the effects of molecular chirality on the supramolecular packing and to understand the impacts of the chiral differences on the self-assembly behavior (Figure 1). All molecules were synthesized according to the solid-phase peptide synthesis method, and the purities of the molecules were determined by LC-MS (Figures S1-S4).

The hierarchal self-assembly of L-VVHH, D-VVHH, L-FFHH, and D-FFHH is controlled by the hydrophobic alkyl tail, the peptide backbones with different hydrogen-bonding abilities, and the hydrophilic double-His headgroup. The $\mathrm{pH}$ under self- 

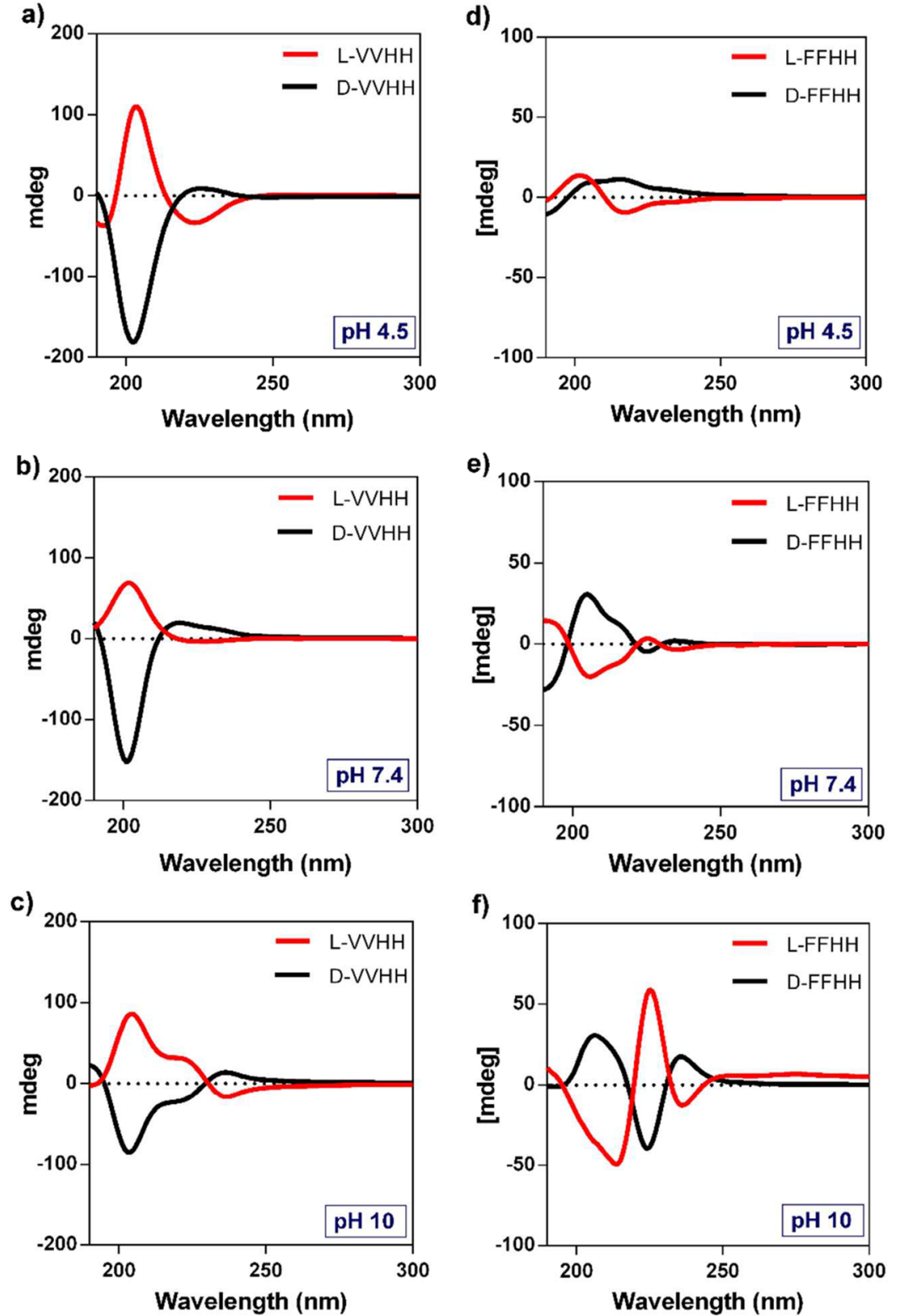

Figure 2. CD spectra of $0.5 \mathrm{mM}$ L-VVHH, D-VVHH, L-FFHH, and D-FFHH at $\mathrm{pH}(\mathrm{a}, \mathrm{d}) 4.5,(\mathrm{~b}, \mathrm{e}) 7.4$, and $(\mathrm{c}, \mathrm{f}) 10$ in water.

assembly conditions was determined according to the $\mathrm{p} K_{\mathrm{a}}$ value of the imidazole ring in the His residues, which is approximately 6.5. ${ }^{55}$ Below this $\mathrm{pH}$ (e.g., in slightly acidic conditions, $\mathrm{pH} 4.5$ ), the side chains of His are protonated, and hydrophilic His residues solubilize the PA molecules in water. The solvophobic interactions ${ }^{56,57}$ enhanced by hydrogen bonding between the $\beta$-sheet-forming residues ${ }^{27}$ and cation $-\pi$ interactions of the His residues facilitate the supramolecular organization of the PAs under acidic conditions. At physiological $\mathrm{pH}$, the imidazole ring can be in either the neutral or positively charged form, ${ }^{58}$ and the molecules are able to self-assemble into supramolecular nanostructures with the help of cation $-\pi$, hydrogen-bonding, and $\pi-\pi$ interactions. The self-assembly is also assisted under basic conditions because the neutral imidazole ring can behave as both a hydrogen donor and a hydrogen acceptor $^{58}$ and can form extensive hydrogen-bonding and $\pi-\pi$ stacking arrays. To reveal the aggregation behavior of the PAs, the critical aggregation concentrations (CACs) of the molecules were first studied using a Nile Red assay. ${ }^{59}$ The CACs of the PAs were determined as being higher than approximately $1 \mu \mathrm{M}$ in water (Figure S5). Hence, all structural studies were carried out above this concentration to obtain histidine-functionalized PA assemblies under slightly acidic ( $\mathrm{pH} 4.5)$, neutral $(\mathrm{pH} 7.4)$, and basic ( $\mathrm{pH} \mathrm{10)}$ conditions.

Structural Characterization of the PA Assemblies. The secondary structures of the L-VVHH, D-VVHH, L-FFHH, and $\mathrm{D}-\mathrm{FFHH}$ assemblies were characterized using circular dichroism (CD). The typical $\beta$-sheet secondary-structure organization shows a characteristic CD spectrum with maximum and minimum peaks at about 195 and $216 \mathrm{~nm}$, respectively. ${ }^{60}$ At $\mathrm{pH} 4.5$, the CD spectrum of $\mathrm{L}-\mathrm{VVHH}$ revealed a twisted $\beta$ sheet secondary structure with red-shifted maximum and minimum peaks at about 205 and $224 \mathrm{~nm}$, respectively, due to the slight distortion from perfect $\beta$-sheets (Figure 2a). ${ }^{61,62}$ In addition, the $\mathrm{CD}$ spectrum of $\mathrm{D}-\mathrm{VVHH}$ showed mirror-image symmetry with respect to that of $\mathrm{L}-\mathrm{VVHH}$ because of chiral inversion (Figure 2a). The position of the positively charged 

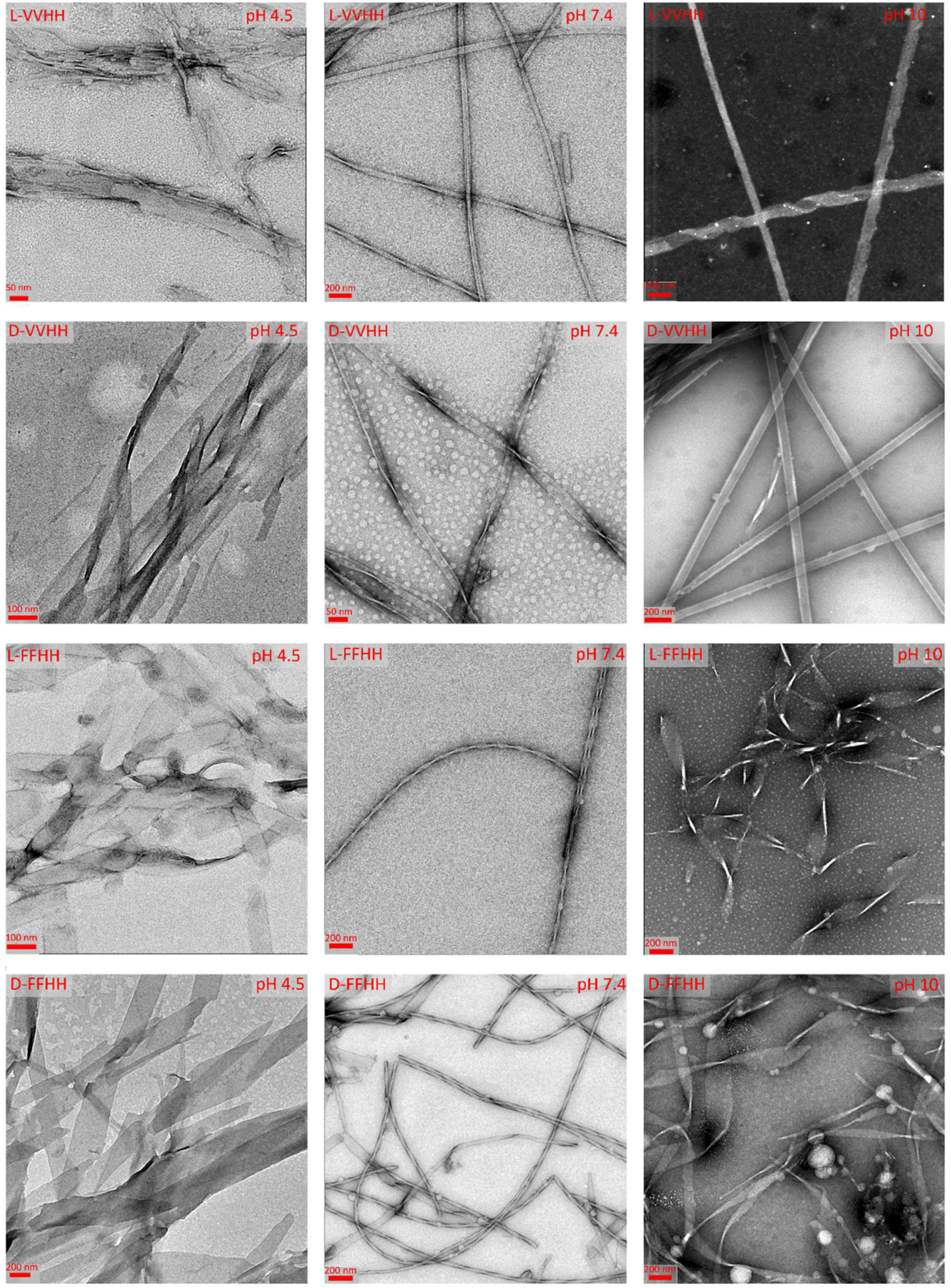

Figure 3. TEM images of the supramolecular L-VVHH, D-VVHH, L-FFHH, and D-FFHH nanostructures at different $\mathrm{pH}$ values.

aromatic His residues in the $\mathrm{L}-$ and $\mathrm{D}-\mathrm{VVHH}$ assemblies could change hydrogen-bonding energies between the residues and prevent the formation of rigid $\beta$-sheets at $\mathrm{pH} 4.5$. Although the CD spectrum of L-VVHH was similar to that of the L-FFHH assembly (Figure 2d), a decrease in CD signal was observed because of differences in the solubilities of the PA assemblies resulting from the enhanced aromaticity of L-FFHH compared to $\mathrm{L}-\mathrm{VVHH}$ at $\mathrm{pH}$ 4.5. On the other hand, mirror-image symmetry was not observed in the CD spectrum of D-FFHH compared to that of L-FFHH (Figure 2d). The CD signal depends on the concentration of the assemblies, and slight differences in the concentrations of the molecules could result in the non-mirror-image symmetry due to experimental errors.

At $\mathrm{pH} 7.4$, the $\mathrm{L}$ - and $\mathrm{D}-\mathrm{VVHH}$ assemblies preserved their twisted $\beta$-sheet organization with mirror-image symmetry (Figure $2 \mathrm{~b}$ ). However, the secondary structure of the $\mathrm{L}$ - and D-FFHH assemblies changed dramatically from twisted $\beta$-sheet to superhelical peptide assemblies. ${ }^{63}$ The $\mathrm{CD}$ spectrum of $\mathrm{L}^{-}$ FFHH exhibited a maximum at $195 \mathrm{~nm}$ and two minima at about 207 and $217 \mathrm{~nm}$, indicating helical organization of the 


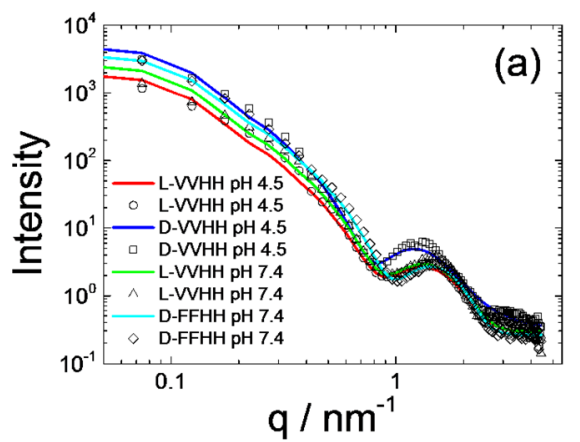

(c)

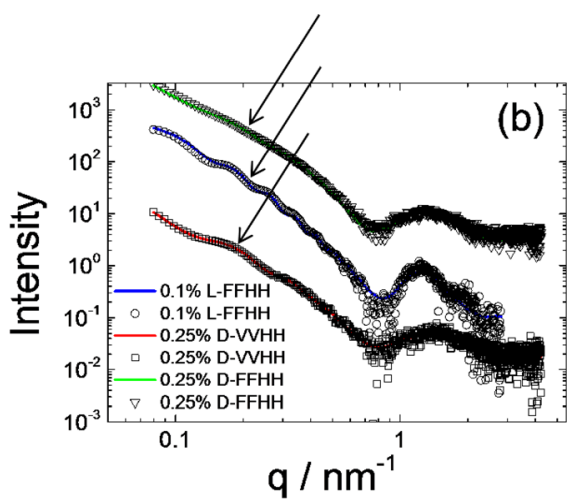

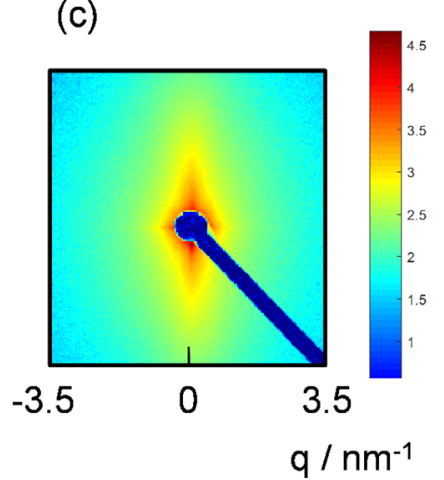

Figure 4. SAXS analysis. (a) Representative data for nanotape-forming samples at $\mathrm{pH} 4.5$ and 7.4. Data (open symbols) were fitted to a bilayer form factor model in each case (solid lines). Fit parameters are listed in Table S1. (b) Nanotube-forming samples at pH 10. Data (open symbols) were fitted to a nanotube form factor model in each case (red lines). Fit parameters are listed in Table S2. Data were multiplied by a factor of 0.1 for D$\mathrm{VVHH}$ and by a factor of 10 for D-FFHH for ease in visualization. The arrows highlight oscillations due to hollow nanotube scattering. (c) Example of an oriented SAXS pattern from $0.25 \mathrm{wt} \%$ sample L-VVHH. The intensity is plotted on a logarithmic scale.

molecules $^{64,65}$ (Figure 2e). The reason for this change is attributed to enhanced $\pi-\pi$ stacking of neutral $-\mathrm{HH}-$ and $-\mathrm{FF}-$ residues of the PAs at $\mathrm{pH}$ 7.4. The potential face-to-face or face-to-edge orientation of the aromatic groups ${ }^{58}$ of $\mathrm{L}$ - and DFFHH molecules through the PA assemblies resulted in helical organization under these conditions. Moreover, the observation of the two additional peaks at about 225 and $240 \mathrm{~nm}$ also pointed to an increase of the $\pi-\pi$ interactions between the $L$ and D-FFHH molecules at $\mathrm{pH} 7.4 .^{66}$ On the other hand, this phenomenon was not observed in the CD spectra of the $\mathrm{L}$ - and $\mathrm{D}-\mathrm{VVHH}$ assemblies because $-\mathrm{VV}-$ residues lack $\pi-\pi$ stacking capabilities and have different hydrogen-bonding properties at $\mathrm{pH}$ 7.4. This difference prevented the possible $\pi-\pi$ stacking of the His residues and made the twisted $\beta$-sheet organization favorable for the L- and $\mathrm{D}-\mathrm{VVHH}$ assemblies at $\mathrm{pH} 7.4$ (Figure 2b).

Under basic conditions, complete neutralization of the His residues resulted in increase of the $\pi-\pi$ interactions and extensive hydrogen bonding for all groups of the PA assemblies. Although aromatic interactions were not observed in the $\mathrm{CD}$ spectra of $\mathrm{L}^{-}$and $\mathrm{D}-\mathrm{VVHH}$ assemblies at $\mathrm{pH} 7.4$, the planar orientation of the neutral His residues under basic conditions without the need for extra aromatic residues led to the formation of $\pi-\pi$ stacking interactions within the L- and DVVHH assemblies. Two additional peaks at about 225 and 240 $\mathrm{nm}$ in the CD spectra of the L- and $\mathrm{D}-\mathrm{VVHH}$ assemblies indicated an enhanced aromaticity with twisted $\beta$-sheet secondary-structure organization (Figure $2 \mathrm{c}$ ). On the other hand, the L- and D-FFHH assemblies showed helical organization with an increase in the CD signals at about 225 and $240 \mathrm{~nm}$, pointing to enhanced aromaticity (Figure 2f).
All PA structures were imaged by TEM at $\mathrm{pH} 4.5,7.4$, and 10 (Figure 3). At $\mathrm{pH} 4.5$, sheetlike nanostructures were observed in the TEM images of all PAs with L or D chirality. Increasing $\mathrm{pH}$ resulted in the formation of twisted nanostructures instead of sheetlike arrangements because of the reduced charge on the imidazole ring in the His residues enhancing the packing of the molecules into ordered supramolecular assemblies. Under basic conditions, the imidazole groups were completely neutralized, and $\pi-\pi$ interactions dominated the supramolecular organization. Hence, the L-VVHH and D-VVHH PA molecules formed well-organized nanotubes because of the multilayer molecular packing of the building blocks. Although wellorganized nanotube formation could not be observed in the TEM images of the L-FFHH and D-FFHH PA molecules at $\mathrm{pH}$ 10 because of the sample preparation, atomic force microscopy (AFM) images of the L-FFHH and D-FFHH assemblies clearly showed nanotube organization at $\mathrm{pH} 10$ (Figure S7).

Prior to the nanotube formation at $\mathrm{pH} \mathrm{10,} \mathrm{the} \mathrm{PAs} \mathrm{initially}$ could form sheetlike nanostructures because of the positive charges on the His residues at $\mathrm{pH}$ 4.5. The charge screening resulting from the increase in $\mathrm{pH}$ promoted the $\pi-\pi$ interactions and resulted in the formation of twisted PA nanostructures at $\mathrm{pH} 7.4$ (Figure 3). Whereas twisting was observed in the TEM images of all molecules at this $\mathrm{pH}$, the Land $\mathrm{D}-\mathrm{FFHH}$ assemblies showed more well-ordered twisting behavior than the $\mathrm{L}^{-}$and $\mathrm{D}-\mathrm{VVHH}$ nanostructures. This mechanism was also analyzed by AFM imaging, and the nanotube formation at $\mathrm{pH} 10$ was observed in the AFM images of all PA assemblies (Figures S6 and S7).

SAXS analysis provides information on nanostructure shape and dimensions in solution. The scattering intensity profiles of 
(a)

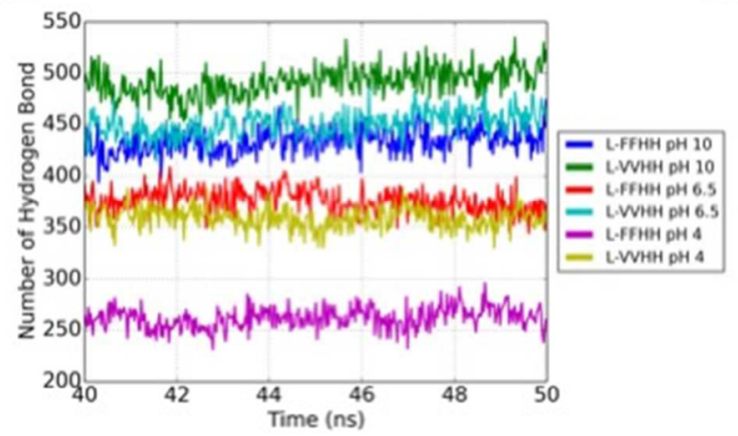

(b)

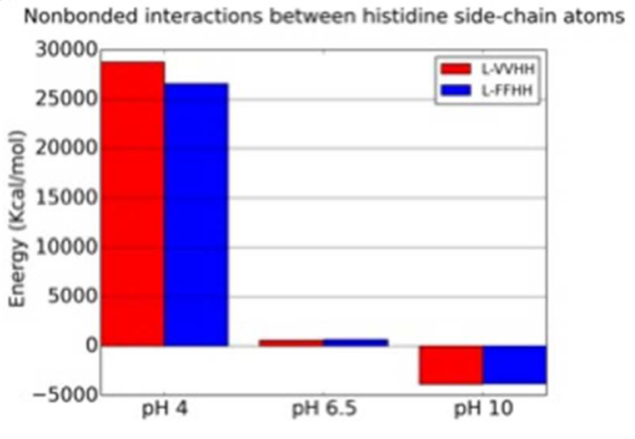

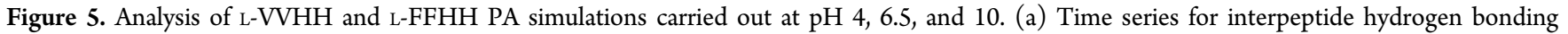
between PA molecules. A hydrogen bond is assumed to form when the distance between the donor and acceptor atoms is less than $3.5 \AA$ and the donor-hydrogen-acceptor angle is greater than $150^{\circ}$. (b) Average of the nonbonded interaction energies between histidine side-chain atoms for the last $10 \mathrm{~ns}$ of the simulations.

the PA assemblies were collected at different $\mathrm{pH}$ values (Figure 4a). The data for all samples, with two exceptions, were fitted to model form-factor profiles corresponding to bilayer structures. Such a model is consistent with a tape nanostructure based on a bilayer form factor that represents the electron density by three Gaussians: two positive Gaussians representing surfaces and an inner Gaussian representing the "core". This model, used in several previous works, ${ }^{67,68}$ is that of Pabst et al. ${ }^{69}$ The fitting was done using SASfit. ${ }^{70}$ The fit parameters are listed in Table S1. The determined "layer thicknesses" are in the range of $2.7-3.8 \mathrm{~nm}$ and indicate the presence of bilayers of the molecules. In some cases, the form-factor maximum has elements of a Bragg peak, suggesting the presence of multiple layers. The estimated length of a tetrapeptide in an antiparallel $\beta$-sheet configuration is $4 \times 0.34 \mathrm{~nm}=1.36 \mathrm{~nm}$. The obtained spacings suggest the presence of bilayers with hydrophobic VV or FF residues in the interior and $\mathrm{HH}$ residues on the exterior.

The samples at $\mathrm{pH} 10$ showed a different form factor, with additional features associated with the closure of bilayer ribbons into nanotubes. The additional features are oscillations at low $q$, highlighted in Figure 4b. These data were fitted to a form factor that combines the bilayer Gauss model with a hollow-cylinder form factor to approximate the form factor of a nanotube containing a bilayer wall structure. The fit parameters are listed in Table S2. SAXS confirmed nanotube formation at $\mathrm{pH} 10$. TEM images of the PA assemblies (Figure 3) also showed ribbon (unwrapped nanotube) and nanotube structures at this $\mathrm{pH}$ value. Partially unwrapped nanotubes might be an artifact of the method for preparing TEM samples, which involves the drying of samples. The nanotube wall thickness from the fit is larger than the bilayer thickness obtained for the nanotapeforming samples. The values obtained indicate that the nanotube walls comprise two bilayers. The SAXS data indicate a higher nanotube radius for L-FFHH, which also had a higher viscosity and required dilution to $0.1 \mathrm{wt} \%$ from $0.25 \mathrm{wt} \%$ for it to flow in the SAXS capillary. A sample of L-VVHH was also viscous. This suggests a higher density or length of nanotubes in the L- $x x \mathrm{HH}$ samples at $\mathrm{pH} 10$ compared to the $\mathrm{D}-x x \mathrm{HH}$ samples. For some samples, a notable orientation of the $2 \mathrm{D}$ SAXS patterns was observed because of the alignment of the peptide tapes or nanotubes under flow into the SAXS capillary. A typical SAXS pattern from an oriented sample is shown in Figure 4c.

Simulation of PA Self-Assembly. To investigate the molecular organization and interactions between the molecules, we performed all-atom explicit-solvent molecular dynamics simulations of L-VVHH, D-VVHH, L-FFHH, and D-FFHH assemblies. $\mathrm{H}$-bond analysis for the simulations of $\mathrm{L}-\mathrm{VVHH}$ and $\mathrm{L}-\mathrm{FFHH}$ PAs under different $\mathrm{pH}$ conditions are shown in Figure 5a. As the $\mathrm{pH}$ increases, the H-bond-forming ability of both L-VVHH and L-FFHH increases. Similar behavior was also observed for D-VVHH and D-FFHH assemblies (Figure S9). The change in hydrogen-bonding capacity of the PAs under different $\mathrm{pH}$ conditions could stem from the interactions between histidine residues. At $\mathrm{pH} 4$, all of the histidine residues are protonated, which results in high repulsion between histidine side-chain atoms (Figure 5b). At $\mathrm{pH} 6.5$, where the histidine residues are partially charged, repulsive interactions between charged side chains are balanced by attractive interactions such as $\pi-\pi$, cation $-\pi$, and hydrogen-bond interactions. At $\mathrm{pH} 10$, the interactions between histidine side chains become attractive, because all side chains are in neutral form.

Moreover, H-bond analyses also showed that FFHH PAs contain fewer interpeptide hydrogen bonds compared to VVHH. The smaller numbers of $\mathrm{H}$-bonds in FFHH PAs could give rise to an increase in the twisting of FFHH PA nanostructures compared to the twisting of VVHH PAs. To elucidate this behavior, we calculated nonbonded interactions between phenylalanine and valine side chains (Figure S10). Phenylalanine side chains in L-FFHH PAs have more attractive interactions than valine side chains in L-VVHH PAs. However, phenylalanine side chains have high repulsive van der Waals interactions, whereas valine side chains have attractive van der Waals interactions (Figure S10). Overall, phenylalanine side chains have repulsive nonbonded interactions, and valine side chains have attractive nonbonded interactions. Thus, steric hindrance between phenylalanine residues could play a significant role in determining the morphology of PA nanostructures.

An analysis of $\mathrm{H}$-bond properties depending on the molecular chirality of the PA molecules was also performed under acidic ( $\mathrm{pH} 4)$, slightly neutral ( $\mathrm{pH}$ 6.5), and basic conditions ( $\mathrm{pH} \mathrm{10)}$ (Figure S9). L-PA molecules tend to form higher numbers of $\mathrm{H}$-bonds compared to D-PA molecules depending on $\mathrm{pH}$ increase (Figures S9 and S11). This observation could be related to the differences of the conformations of $\mathrm{D}$ - and L-amino acids in the peptide backbone, which can affect noncovalent interactions between the molecules. In fact, several studies showed that the $\mathrm{D}$-form of 
amino acids could alter $\beta$-sheet-forming ability of PA nanofibers depending on the amino acid chirality of the molecules. ${ }^{71}$

Self-Assembly of the Chiral Mixtures. To systematically analyze the effects of molecular chirality on the self-assembly behavior of the PAs, chiral mixtures consisting of both D$\mathrm{VVHH}$ and $\mathrm{L}-\mathrm{VVHH}$ at different molar ratios were prepared at $\mathrm{pH} 10$ according to the protocol given in detail in the Experimental Section. The CD spectra of $100 \% \mathrm{~L}-\mathrm{VVHH}$ and $100 \%$ D-VVHH showed a nearly symmetric Cotton effect. This reflects the opposite handedness of packing in the nanostructures at $\mathrm{pH} 10$. According to secondary-structure analysis of the chiral mixtures in Figure 6, an increase in the D-VVHH amount

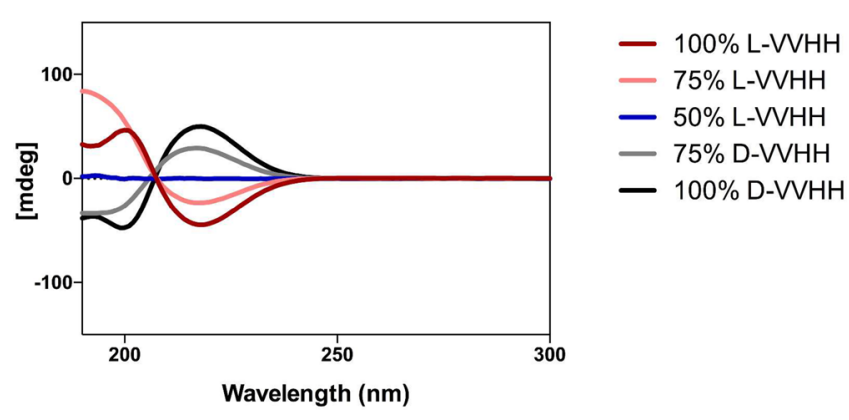

Figure 6. CD spectra of chiral mixtures of $0.5 \mathrm{mM}$ L-VVHH with DVVHH prepared at different molar ratios.

in the $\mathrm{L}-\mathrm{VVHH}$ solution decreased the $\beta$-sheet $\mathrm{CD}$ signal because of packing constraints. This orientation change caused a decrease in the extent of nanoscale chiral organization. A further increment in the $\mathrm{D}-\mathrm{VVHH}$ amount above the $\mathrm{L}-\mathrm{VVHH}$ amount led to a $\beta$-sheet signal that was mirror-symmetric with respect to tht of $\mathrm{L}-\mathrm{VVHH}$ (Figure 6). Racemic mixtures not only showed nearly zero $\mathrm{CD}$ signal but also formed twisted nanostructures rather than nanotubes (Figure 7). SAXS data from selected mixtures studied (at $\mathrm{pH} 10$ ) can be fitted to a model for bilayers consistent with the twisted nanotape structures observed by TEM. The SAXS data along with model form-factor fits are shown in Figure 8. The fit parameters are listed in Table S3.

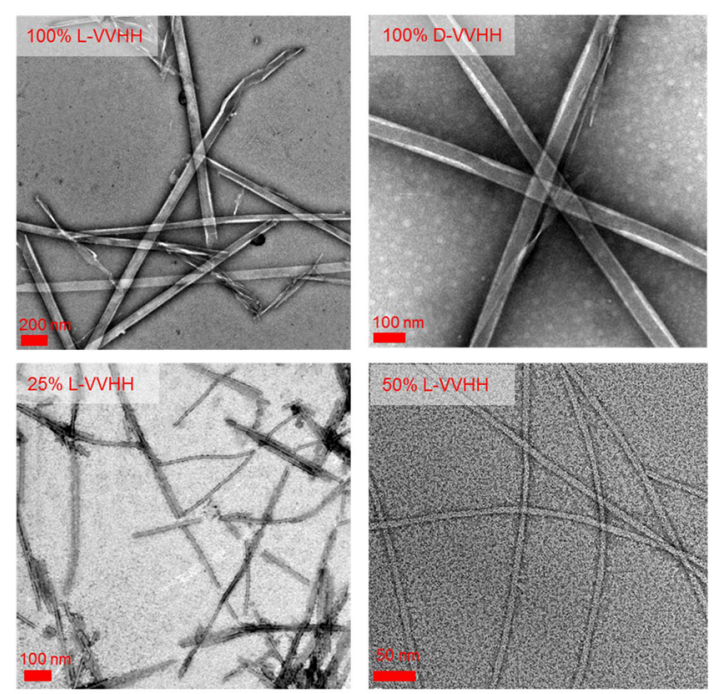

Figure 7. TEM images of the supramolecular nanostructures prepared with chiral mixtures of $\mathrm{L}-\mathrm{VVHH}$ with $\mathrm{D}-\mathrm{VVHH}$ prepared at different molar ratios.

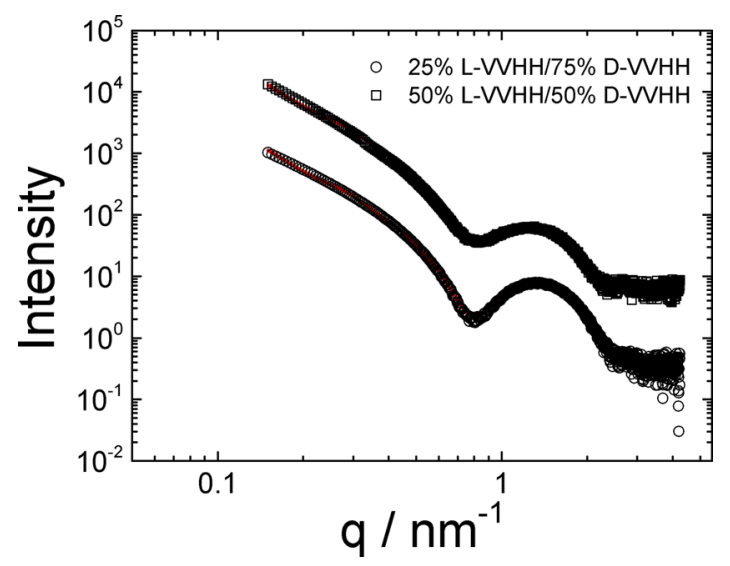

Figure 8. SAXS data for mixtures as indicated. Data (open symbols) were fitted to a bilayer form factor model in each case (red lines). Fit parameters are listed in Table S3. Data were multiplied by a factor of 10 for $50 \% \mathrm{~L}-\mathrm{VVHH} / 50 \% \mathrm{D}-\mathrm{VVHH}$ for ease in visualization.

Simulation of the Self-Assembly of the Chiral Mixtures. In addition to simulation studies of individual LVVHH, D-VVHH, L-FFHH, and D-FFHH assemblies, the noncovalent interactions between the chiral mixtures were also analyzed through molecular dynamics simulations performed for $50 \mathrm{~ns}$. H-bond analysis of these simulations suggests that the pure L-PA systems have more hydrogen bonds than mixtures of L-PA and D-PAs (Figure S11). As the amount of D-PA in L-PA systems increases, the hydrogen-bonding capacity decreases. However, when the system is composed of only D-PAs, then the hydrogen bonding tends to increase. Pure L-PA and D-PA nanostructures tend to have higher hydrogen-bonding abilities than nanostructures composed of racemic mixtures of these PAs.

\section{CONCLUSIONS}

In summary, the hierarchical assemblies of the His-functionalized PAs and their chiral mixtures were characterized using different techniques and molecular dynamics simulations to reveal supramolecular organization of the PA assemblies and their chiral mixtures. It was shown that the supramolecular organization of His-functionalized PAs is controlled by the solution $\mathrm{pH}$ and the molecular chirality of the building blocks. Whereas self-assembly of the PAs into nanosheets is driven by solvophobic interactions under acidic conditions, the deprotonation of the His residues due to a $\mathrm{pH}$ increase changes the self-assembly behavior and results in the formation of stable twisted nanostructures at neutral $\mathrm{pH}$. Moreover, an increase in solution basicity facilitates the transition from twisted nanostructures into closed nanotubes through an enhanced hydrogen-bonding capacity and $\pi-\pi$ stacking of the imidazole ring at $\mathrm{pH} 10$.

\section{ASSOCIATED CONTENT}

S Supporting Information

The Supporting Information is available free of charge on the ACS Publications website at DOI: 10.1021/acs.langmuir.7b01266.

LC-MS and CAC results; supporting AFM, TEM, and SEM images of the PA assemblies; details of SAXS data fitting with estimated structural parameters; and results of simulation studies (PDF) 


\section{AUTHOR INFORMATION}

\section{Corresponding Author}

*E-mail: mguler@uchicago.edu.

\section{ORCID}

Valeria Castelletto: 0000-0002-3705-0162

Ian W. Hamley: 0000-0002-4549-0926

Mustafa O. Guler: 0000-0003-1168-202X

\section{Notes}

The authors declare no competing financial interest.

\section{ACKNOWLEDGMENTS}

This work was partially supported by grants from TUBITAK (114Z728), TUBA-GEBIP, and FP7Marie Curie IRG. M.H.K. and G.C. are supported by TUBITAK-BIDEB 2210-C and 2211-C fellowships. The simulations reported in this work were performed at TUBITAK ULAKBIM, High Performance and Grid Computing Center (TRUBA resources). We thank Mr. M. Guler for help in TEM imaging. I.W.H. is grateful to EPSRC (U.K.) for Grant EP/L020599/1. We thank the ESRF for the award of bioSAXS beamtime on BM29 (ref MX-1769), Gabriele Giachin for assistance with the measurements, and Jerome Kieffer for data remasking and rebinning.

\section{REFERENCES}

(1) Whitesides, G. M.; Grzybowski, B. Self-assembly at all scales. Science 2002, 295 (5564), 2418-2421.

(2) Gobre, V. V.; Tkatchenko, A. Scaling laws for van der Waals interactions in nanostructured materials. Nat. Commun. 2013, 4, 2341.

(3) Gao, H.-Y.; Wagner, H.; Held, P. A.; Du, S.; Gao, H.-J.; Studer, A.; Fuchs, H. In-plane Van der Waals interactions of molecular selfassembly monolayer. Appl. Phys. Lett. 2015, 106 (8), 081606.

(4) Shen, Z.; Wang, T.; Liu, M. H-bond and $\pi-\pi$ stacking directed self-assembly of two-component supramolecular nanotubes: tuning length, diameter and wall thickness. Chem. Commun. 2014, 50 (17), 2096-2099.

(5) Hoeben, F. J.; Jonkheijm, P.; Meijer, E.; Schenning, A. P. About supramolecular assemblies of $\pi$-conjugated systems. Chem. Rev. 2005, 105 (4), 1491-1546.

(6) Sherrington, D. C.; Taskinen, K. A. Self-assembly in synthetic macromolecular systems multiple hydrogen bonding interactions. Chem. Soc. Rev. 2001, 30 (2), 83-93.

(7) Swiegers, G. F.; Malefetse, T. J. New self-assembled structural motifs in coordination chemistry. Chem. Rev. 2000, 100 (9), 34833538 .

(8) Zhang, L.; Wang, X.; Wang, T.; Liu, M. Tuning Soft Nanostructures in Self-assembled Supramolecular Gels: From Morphology Control to Morphology-Dependent Functions. Small 2015, 11 (9-10), 1025-1038.

(9) Debnath, S.; Roy, S.; Ulijn, R. V. Peptide nanofibers with dynamic instability through nonequilibrium biocatalytic assembly. $J$. Am. Chem. Soc. 2013, 135 (45), 16789-16792.

(10) Cinar, G.; Orujalipoor, I.; Su, C.-J.; Jeng, U. S.; Ide, S.; Guler, M. O. Supramolecular Nanostructure Formation of Coassembled Amyloid Inspired Peptides. Langmuir 2016, 32 (25), 6506-6514.

(11) Mammadov, R.; Cinar, G.; Gunduz, N.; Goktas, M.; Kayhan, H.; Tohumeken, S.; Topal, A. E.; Orujalipoor, I.; Delibasi, T.; Dana, A.; Ide, S.; Tekinay, A. B.; Guler, M. O. Virus-like nanostructures for tuning immune response. Sci. Rep. 2015, 5, 16728.

(12) Mumcuoglu, D.; Sardan Ekiz, M.; Gunay, G.; Tekinay, T.; Tekinay, A. B.; Guler, M. O. Cellular Internalization of Therapeutic Oligonucleotides by Peptide Amphiphile Nanofibers and Nanospheres. ACS Appl. Mater. Interfaces 2016, 8 (18), 11280-11287.

(13) Kol, N.; Adler-Abramovich, L.; Barlam, D.; Shneck, R. Z.; Gazit, E.; Rousso, I. Self-assembled peptide nanotubes are uniquely rigid bioinspired supramolecular structures. Nano Lett. 2005, 5 (7), 13431346.

(14) Shimizu, T.; Kameta, N.; Ding, W.; Masuda, M. Supramolecular Self-Assembly into Biofunctional Soft Nanotubes: From Bilayers to Monolayers. Langmuir 2016, 32 (47), 12242-12264.

(15) Hamley, I. W. Peptide nanotubes. Angew. Chem., Int. Ed. 2014, 53 (27), 6866-6881.

(16) Xie, Y.; Wang, X.; Huang, R.; Qi, W.; Wang, Y.; Su, R.; He, Z. Electrostatic and aromatic interaction-directed supramolecular selfassembly of a designed Fmoc-tripeptide into helical nanoribbons. Langmuir 2015, 31 (9), 2885-2894.

(17) Zhang, S.; Marini, D. M.; Hwang, W.; Santoso, S. Design of nanostructured biological materials through self-assembly of peptides and proteins. Curr. Opin. Chem. Biol. 2002, 6 (6), 865-871.

(18) Ekiz, M. S.; Cinar, G.; Khalily, M. A.; Guler, M. O. Selfassembled peptide nanostructures for functional materials. Nanotechnology 2016, 27 (40), 402002.

(19) Wang, J.; Liu, K.; Xing, R.; Yan, X. Peptide self-assembly: thermodynamics and kinetics. Chem. Soc. Rev. 2016, 45 (20), 55895604.

(20) Hartgerink, J. D.; Beniash, E.; Stupp, S. I. Peptide-amphiphile nanofibers: A versatile scaffold for the preparation of self-assembling materials. Proc. Natl. Acad. Sci. U. S. A. 2002, 99 (8), 5133-5138.

(21) Zhao, X.; Pan, F.; Xu, H.; Yaseen, M.; Shan, H.; Hauser, C. A.; Zhang, S.; Lu, J. R. Molecular self-assembly and applications of designer peptide amphiphiles. Chem. Soc. Rev. 2010, 39 (9), 34803498.

(22) Hu, Y.; Lin, R.; Patel, K.; Cheetham, A. G.; Kan, C.; Cui, H. Spatiotemporal control of the creation and immolation of peptide assemblies. Coord. Chem. Rev. 2016, 320-321, 2-17.

(23) Paramonov, S. E.; Jun, H.-W.; Hartgerink, J. D. Self-assembly of peptide- amphiphile nanofibers: the roles of hydrogen bonding and amphiphilic packing. J. Am. Chem. Soc. 2006, 128 (22), 7291-7298.

(24) Ortony, J. H.; Newcomb, C. J.; Matson, J. B.; Palmer, L. C.; Doan, P. E.; Hoffman, B. M.; Stupp, S. I. Internal dynamics of a supramolecular nanofibre. Nat. Mater. 2014, 13 (8), 812-816.

(25) Dasgupta, A. Exploring architectures at the nanoscale: the interplay between hydrophobic twin lipid chains and head groups of designer peptide amphiphiles in the self-assembly process and application. Soft Matter 2016, 12 (19), 4352-4360.

(26) Löwik, D. W. P. M.; Garcia-Hartjes, J.; Meijer, J. T.; van Hest, J. C. M. Tuning Secondary Structure and Self-Assembly of Amphiphilic Peptides. Langmuir 2005, 21 (2), 524-526.

(27) Garifullin, R.; Guler, M. O. Supramolecular chirality in selfassembled peptide amphiphile nanostructures. Chem. Commun. 2015, 51 (62), 12470-12473.

(28) Zhang, S.; Greenfield, M. A.; Mata, A.; Palmer, L. C.; Bitton, R.; Mantei, J. R.; Aparicio, C.; de la Cruz, M. O.; Stupp, S. I. A selfassembly pathway to aligned monodomain gels. Nat. Mater. 2010, 9 (7), 594-601.

(29) Miravet, J. F.; Escuder, B.; Segarra-Maset, M. D.; Tena-Solsona, M.; Hamley, I. W.; Dehsorkhi, A.; Castelletto, V. Self-assembly of a peptide amphiphile: transition from nanotape fibrils to micelles. Soft Matter 2013, 9 (13), 3558-3564.

(30) Matson, J. B.; Navon, Y.; Bitton, R.; Stupp, S. I. LightControlled Hierarchical Self-Assembly of Polyelectrolytes and Supramolecular Polymers. ACS Macro Lett. 2015, 4 (1), 43-47.

(31) Muraoka, T.; Cui, H.; Stupp, S. I. Quadruple Helix Formation of a Photoresponsive Peptide Amphiphile and Its Light-Triggered Dissociation into Single Fibers. J. Am. Chem. Soc. 2008, 130 (10), 2946-2947.

(32) Tsonchev, S.; Niece, K. L.; Schatz, G. C.; Ratner, M. A.; Stupp, S. I. Phase Diagram for Assembly of Biologically-Active Peptide Amphiphiles. J. Phys. Chem. B 2008, 112 (2), 441-447.

(33) Korevaar, P. A.; Newcomb, C. J.; Meijer, E. W.; Stupp, S. I. Pathway Selection in Peptide Amphiphile Assembly. J. Am. Chem. Soc. 2014, 136 (24), 8540-8543.

(34) Qin, S.-Y.; Jiang, H.-F.; Peng, M.-Y.; Lei, Q.; Zhuo, R.-X.; Zhang, X.-Z. Adjustable nanofibers self-assembled from an irregular 
conformational peptide amphiphile. Polym. Chem. 2015, 6 (4), 519524.

(35) Chen, Y.; Gan, H. X.; Tong, Y. W. pH-Controlled Hierarchical Self-Assembly of Peptide Amphiphile. Macromolecules 2015, 48 (8), 2647-2653.

(36) da Silva, R. M. P.; van der Zwaag, D.; Albertazzi, L.; Lee, S. S.; Meijer, E. W.; Stupp, S. I. Super-resolution microscopy reveals structural diversity in molecular exchange among peptide amphiphile nanofibres. Nat. Commun. 2016, 7, 11561.

(37) Fleming, S.; Debnath, S.; Frederix, P. W. J. M.; Tuttle, T.; Ulijn, R. V. Aromatic peptide amphiphiles: significance of the Fmoc moiety. Chem. Commun. 2013, 49 (90), 10587-10589.

(38) Tao, K.; Levin, A.; Adler-Abramovich, L.; Gazit, E. Fmocmodified amino acids and short peptides: simple bio-inspired building blocks for the fabrication of functional materials. Chem. Soc. Rev. 2016, 45 (14), 3935-3953.

(39) Ma, M.; Kuang, Y.; Gao, Y.; Zhang, Y.; Gao, P.; Xu, B. Aromatic-Aromatic Interactions Induce the Self-Assembly of Pentapeptidic Derivatives in Water To Form Nanofibers and Supramolecular Hydrogels. J. Am. Chem. Soc. 2010, 132 (8), 27192728.

(40) Hamley, I. W.; Dehsorkhi, A.; Castelletto, V.; Furzeland, S.; Atkins, D.; Seitsonen, J.; Ruokolainen, J. Reversible helical unwinding transition of a self-assembling peptide amphiphile. Soft Matter 2013, 9 (39), 9290-9293.

(41) Fleming, S.; Ulijn, R. V. Design of nanostructures based on aromatic peptide amphiphiles. Chem. Soc. Rev. 2014, 43 (23), 81508177.

(42) Frisch, H.; Besenius, P. pH-Switchable Self-Assembled Materials. Macromol. Rapid Commun. 2015, 36 (4), 346-363.

(43) Lin, B. F.; Megley, K. A.; Viswanathan, N.; Krogstad, D. V.; Drews, L. B.; Kade, M. J.; Qian, Y.; Tirrell, M. V. pH-responsive branched peptide amphiphile hydrogel designed for applications in regenerative medicine with potential as injectable tissue scaffolds. $J$. Mater. Chem. 2012, 22 (37), 19447-19454.

(44) Moyer, T. J.; Finbloom, J. A.; Chen, F.; Toft, D. J.; Cryns, V. L.; Stupp, S. I. $\mathrm{pH}$ and Amphiphilic Structure Direct Supramolecular Behavior in Biofunctional Assemblies. J. Am. Chem. Soc. 2014, 136 (42), 14746-14752.

(45) Castelletto, V.; Cheng, G.; Stain, C.; Connon, C. J.; Hamley, I. W. Self-Assembly of a Peptide Amphiphile Containing L-Carnosine and Its Mixtures with a Multilamellar Vesicle Forming Lipid. Langmuir 2012, 28 (31), 11599-11608.

(46) Castelletto, V.; Hamley, I. W.; Segarra-Maset, M. D.; Gumbau, C. B.; Miravet, J. F.; Escuder, B.; Seitsonen, J.; Ruokolainen, J. Tuning Chelation by the Surfactant-Like Peptide A(6)H Using Predetermined $\mathrm{pH}$ Values. Biomacromolecules 2014, 15 (2), 591-598.

(47) Hamley, I. W.; Kirkham, S.; Dehsorkhi, A.; Castelletto, V.; Adamcik, J.; Mezzenga, R.; Ruokolainen, J.; Mazzuca, C.; Gatto, E.; Venanzi, M.; Placidi, E.; Bilalis, P.; Iatrou, H. Self-Assembly of a Model Peptide Incorporating a Hexa-Histidine Sequence Attached to an Oligo-Alanine Sequence, and Binding to Gold NTA/Nickel Nanoparticles. Biomacromolecules 2014, 15, 3412-3420.

(48) Zengin, A.; Cinar, G.; Guler, M. O. Controlled enzymatic stability and release characteristics of supramolecular chiral peptide amphiphile nanofiber gels. Curr. Appl. Phys. 2017, 17 (5), 785-792.

(49) Tekin, E. D. Molecular dynamics simulations of self-assembled peptide amphiphile based cylindrical nanofibers. RSC Adv. 2015, 5 (82), 66582-66590.

(50) MacKerell, A. D.; Bashford, D.; Bellott, M.; Dunbrack, R. L.; Evanseck, J. D.; Field, M. J.; Fischer, S.; Gao, J.; Guo, H.; Ha, S.; Joseph-McCarthy, D.; Kuchnir, L.; Kuczera, K.; Lau, F. T. K.; Mattos, C.; Michnick, S.; Ngo, T.; Nguyen, D. T.; Prodhom, B.; Reiher, W. E.; Roux, B.; Schlenkrich, M.; Smith, J. C.; Stote, R.; Straub, J.; Watanabe, M.; Wiórkiewicz-Kuczera, J.; Yin, D.; Karplus, M. All-Atom Empirical Potential for Molecular Modeling and Dynamics Studies of Proteins. J. Phys. Chem. B 1998, 102 (18), 3586-3616.

(51) Phillips, J. C.; Braun, R.; Wang, W.; Gumbart, J.; Tajkhorshid, E.; Villa, E.; Chipot, C.; Skeel, R. D.; Kalé, L.; Schulten, K. Scalable molecular dynamics with NAMD. J. Comput. Chem. 2005, 26 (16), $1781-1802$.

(52) Essmann, U.; Perera, L.; Berkowitz, M. L.; Darden, T.; Lee, H.; Pedersen, L. G. A smooth particle mesh Ewald method. J. Chem. Phys. 1995, 103 (19), 8577-8593.

(53) Roe, D. R.; Cheatham, T. E. PTRAJ and CPPTRAJ: Software for Processing and Analysis of Molecular Dynamics Trajectory Data. J. Chem. Theory Comput. 2013, 9 (7), 3084-3095.

(54) Humphrey, W.; Dalke, A.; Schulten, K. VMD: Visual molecular dynamics. J. Mol. Graphics 1996, 14 (1), 33-38.

(55) Liao, S.-M.; Du, Q.-S.; Meng, J.-Z.; Pang, Z.-W.; Huang, R.-B. The multiple roles of histidine in protein interactions. Chem. Cent. J. 2013, 7, 44-44.

(56) Elemans, J. A. A. W.; Rowan, A. E.; Nolte, R. J. M. Mastering molecular matter. Supramolecular architectures by hierarchical selfassembly. J. Mater. Chem. 2003, 13 (11), 2661-2670.

(57) Fu, I. W.; Markegard, C. B.; Nguyen, H. D. Solvent Effects on Kinetic Mechanisms of Self-Assembly by Peptide Amphiphiles via Molecular Dynamics Simulations. Langmuir 2015, 31 (1), 315-324.

(58) Bhattacharyya, R.; Saha, R. P.; Samanta, U.; Chakrabarti, P. Geometry of Interaction of the Histidine Ring with Other Planar and Basic Residues. J. Proteome Res. 2003, 2 (3), 255-263.

(59) Hamsici, S.; Cinar, G.; Celebioglu, A.; Uyar, T.; Tekinay, A. B.; Guler, M. O. Bioactive peptide functionalized aligned cyclodextrin nanofibers for neurite outgrowth. J. Mater. Chem. B 2017, 5 (3), 517524.

(60) Micsonai, A.; Wien, F.; Kernya, L.; Lee, Y.-H.; Goto, Y.; Réfrégiers, M.; Kardos, J. Accurate secondary structure prediction and fold recognition for circular dichroism spectroscopy. Proc. Natl. Acad. Sci. U. S. A. 2015, 112 (24), E3095-E3103.

(61) Pashuck, E. T.; Cui, H.; Stupp, S. I. Tuning supramolecular rigidity of peptide fibers through molecular structure. J. Am. Chem. Soc. 2010, 132 (17), 6041-6046.

(62) Hamley, I. W.; Nutt, D. R.; Brown, G. D.; Miravet, J. F.; Escuder, B.; Rodríguez-Llansola, F. Influence of the Solvent on the Self-Assembly of a Modified Amyloid Beta Peptide Fragment. II. NMR and Computer Simulation Investigation. J. Phys. Chem. B 2010, 114, 940-951.

(63) Mondal, S.; Adler-Abramovich, L.; Lampel, A.; Bram, Y.; Lipstman, S.; Gazit, E. Formation of functional super-helical assemblies by constrained single heptad repeat. Nat. Commun. 2015, 6, 8615 .

(64) Chin, D.-H.; Woody, R. W.; Rohl, C. A.; Baldwin, R. L. Circular dichroism spectra of short, fixed-nucleus alanine helices. Proc. Natl. Acad. Sci. U. S. A. 2002, 99 (24), 15416-15421.

(65) Shepherd, N. E.; Hoang, H. N.; Abbenante, G.; Fairlie, D. P. Single turn peptide alpha helices with exceptional stability in water. J. Am. Chem. Soc. 2005, 127 (9), 2974-2983.

(66) Pasc, A.; Obounou Akong, F.; Cosgun, S.; Gérardin, C. Differences between $\beta$-Ala and Gly-Gly in the design of amino acidsbased hydrogels. Beilstein J. Org. Chem. 2010, 6 (1), 973-977.

(67) Castelletto, V.; Hamley, I. W.; Adamcik, J.; Mezzenga, R.; Gummel, J. Modulating self-assembly of a nanotape-forming peptide amphiphile with an oppositely charged surfactant. Soft Matter 2012, 8 (1), 217-226.

(68) Castelletto, V.; Hamley, I. W.; Whitehouse, C.; Matts, P. J.; Osborne, R.; Baker, E. S. Self-Assembly of Palmitoyl Lipopeptides Used in Skin Care Products. Langmuir 2013, 29 (29), 9149-9155.

(69) Pabst, G.; Rappolt, M.; Amenitsch, H.; Laggner, P. Structural information from multilamellar liposomes at full hydration: Full qrange fitting with high quality X-ray data. Phys. Rev. E: Stat. Phys., Plasmas, Fluids, Relat. Interdiscip. Top. 2000, 62 (3), 4000-4009.

(70) Bressler, I.; Kohlbrecher, J.; Thunemann, A. F. SASfit: a tool for small-angle scattering data analysis using a library of analytical expressions. J. Appl. Crystallogr. 2015, 48 (5), 1587-1598.

(71) Melchionna, M.; Styan, K. E.; Marchesan, S. The Unexpected Advantages of Using D-Amino Acids for Peptide Self- Assembly into Nanostructured Hydrogels for Medicine. Curr. Top. Med. Chem. 2016, 16 (18), 2009-2018. 